\section{Microlithiasis alveolaris pulmonum}

Zusammenfassung: Es wird über einen Fall von pulmonaler alveolärer Mikrolithiasis bei einem 50 -jährigen Patienten berichtet. Die Diagnose wurde mittels transbronchialer Lungenbiopsie histologisch gesichert. Der radiologische sowie der bodyplethysmographische Befund blieben im Verlauf von 4 Jahren unverändert.

Pulmonary Alveolar Microlithiasis: Pulmonary alveolar microlithiasis is a rare disease of unknown aetiology, in which calcium phosphate microliths are deposited bilaterally throughout the lung parenchyma. The total number of reported cases is about 400 . A case of pulmonary alveolar microlithiasis is presented in a 50-year old man for whom the diagnosis was confirmed by histology via transbronchial biopsy. During a 4-year observation period there was no increase of the disease according to $x$ ray investigations and with respect to clinical symptoms.

\section{Einleitung}

Die pulmonale alveoläre Mikrolithiasis ist rar. Rund 400 Fälle sind bis jetzt in der medizinischen Literatur beschrieben worden. Nach den vorliegenden Berichten ist die Krankheit weltweit bei allen Völkern verbreitet. Ein Unterschied in der geographischen Verteilung ist möglich.

In Japan sind bis 1970 insgesamt 60 [1], in der Türkei bis 1993 52 Fälle [2] bekannt geworden. Die Krankheit ist durch Füllung der Alveolen mit Steinen in den Unterfeldern charakterisiert, und bei fortschreitender Erkrankung werden weitere Alveolen befallen, dabei können die Steine die Alveolen bis zu $80 \%$ ausfüllen. Manchmal befinden sie sich in den Alveolärsepten [1,3], gelegentlich aber auch in den Bronchialwänden [4-6]. Mikhailov beschrieb 1954 Mikrolithen sogar auch im Lungeninterstitium [7]. Die meisten gefundenen Steine wiesen einen Durchmesser von 0,2 mm auf. Prakash und Mitarb. [8] fanden in den Alveolen eines 20-jährigen Patienten nach offener Lungenbiopsie Mikrolithen von 0,01 bis $2,8 \mathrm{~mm}$ im Durchmesser. Pankow und Mitarb. [9] berichteten über bis zu $3 \mathrm{~mm}$ große Verdichtungsherde im Röntgenbild, die sich später als Mikrolithen herausstellten. Sie sind rund, oval oder

Pneumologie 2001; 55: 149-151

(c) Georg Thieme Verlag Stuttgart · New York ISSN 0934-8387

\section{H. Gubbawy}

Praxis für Lungen- und Bronchialheilkunde, Neumünster haben manchmal eine unregelmäßige Form und sind konzentrisch beschichtet.

Die Ätiologie ist ungeklärt; es gilt aber als sicher, dass eine Calcium-Phosphor-Stoffwechselstörung vorliegt und Erbfaktoren eine Rolle spielen, etwa die Hälfte der Fälle treten familiär auf. Die Annahme, dass eine Staubexposition, ein Nikotin- oder Schnupftabakabusus mögliche Faktoren für die Genese der Erkrankung darstellen, ist sehr zweifelhaft, da die Erkrankung auch bei Kindern und sogar bei Neugeborenen vorkommt und familiär gehäuft auftritt [10]. In Deutschland sind bis jetzt insgesamt 26 Fälle, 13 männliche und 13 weibliche, bekannt geworden. Über einen zusätzlichen Fall aus meiner Praxis wird berichtet.

\section{Kasuistik}

Ein 50-jähriger Patient hat bis 1985 als Maurer gearbeitet, insgesamt 25 Jahre lang. Seit 1985 ist er im Katasteramt tätig. Im Rahmen der Vorbereitung einer Operation am rechten Knie erfolgte bei ihm eine Röntgenthoraxuntersuchung in einer radiologischen Praxis, wobei multiple kleine Noduli in den Mittel- und Unterfeldern aufgefallen sind, die durch eine Thorax-Computertomographie bestätigt wurden. Auf einer Schirmbildaufnahme von 1985 waren die beschriebenen röntgenologischen Veränderungen nicht erkennbar.

Bei der klinischen Untersuchung waren die Lungen unauffällig. Die Ruhespirometrie und Bodyplethysmographie ergaben keine Hinweise für eine obstruktive oder restriktive Ventilationsstörung. Bronchoskopisch war das Bronchialsystem unauffällig, die histologische Untersuchung der Bronchialschleimhaut (Prof. Dr. Fohlmeister, Neumünster) ergab keinen wesentlichen histologischen Befund.

Die histologische Untersuchung der transbronchialen Lungenbiopsie ergab eine pulmonale alveoläre Mikrolithiasis mit Mikrolithen bis zu einem Durchmesser von 0,2 mm.

Bei der letzten Kontrolluntersuchung im August 1999 blieb der Röntgenthoraxbefund unverändert. Die Ruhespirometrie und Bodyplethysmographie ergaben nach wie vor keinen Anhalt für eine obstruktive oder restriktive Ventilationsstörung. Die Blutgasanalyse zeigte einen normalen Sauerstoffund Kohlendioxidpartialdruck bei ausgeglichenem Säurebasenhaushalt. Die CO-Diffusionskapazität lag im Normbereich. 


\section{Historisches}

Die Erstbeschreibung der pulmonalen alveolären Mikrolithiasis geht auf Harbitz 1918 [11] zurück. Er fand bei einer Autopsie einer 41-jährigen an Arrhythmien, Beinödemen, Zyanose und Dyspnoe verstorbenen Patientin Pleuraergüsse, Aszites, Herzvergrößerung und harte Lungen, die sich wie Steine anfühlten. Die ausgeschnittenen Lungenteile schwammen nicht in Wasser. Die histologische Untersuchung ergab Mikrolithen in den Alveolen.

1933 hat Puhr [12] aus Budapest über einen 49-jährigen an Extremitätenödemen, Herzvergrößerung und Tachyarrhythmie verstorbenen Patienten berichtet. Bei der Sektion fand er schwere und steife Lungen, als ob sie Gipsausgüsse wären. Die ausgeschnittenen Teile sanken in Wasser unter. Bei der histologischen Untersuchung hat er in den erweiterten Lungenalveolen konzentrisch beschichtete Mikrolithen festgestellt. Auf ihn geht die Benennung der Krankheit Mikrolithiasis alveolaris pulmonum zurück.

Aus Solingen haben Landes und Leicher 1948 [13] einen Fall von einem 44-jährigen Patienten, der an Zeichen einer respiratorischen Insuffizienz mit Zyanose und Trommelschlegelfingern ad exitum kam, geschildert. Die Obduktion hat schwere knorpelharte Lungen gezeigt, und die Diagnose einer alveolären Mikrolithiasis konnte histologisch gesichert werden. Die Verfasser haben zum ersten Mal eine chemische Analyse der alveolären Steine durchgeführt.

Lindig hat 1951 [14] zum ersten Mal die Krankheit radiologisch diagnostiziert, und dadurch wurde sie erstmals intravital erkannt. Mikhailov hat als erster 1954 [7] über das Vorkommen der Krankheit bei mehreren Familienmitgliedern berichtet und stellte die Frage der Erbmöglichkeit dieser Erkrankung. Petrányi und Mitarb. stellten 1954 [15] die Diagnose erstmals nach einer Thorakotomie bei einem 8jährigen Kind fest und dadurch wiesen sie nach, dass die Krankheit auch im Kindesalter vorkommt. Sosman und Mitarb. berichteten 1957 [16] ausführlich über die Klinik und Diagnose dieser Erkrankung aus der Weltliteratur.

\section{Klinik}

Mehr als die Hälfte der Patienten weisen bis zur Sicherung der Diagnose keine bronchopulmonale Symptomatik auf. Die meisten Fälle werden zufällig durch eine Routineröntgenthoraxuntersuchung oder im Rahmen einer Untersuchung der Familienmitglieder einer erkrankten Person entdeckt.

In der Frühphase der Erkrankung weicht der Perkussions- und Auskultationsbefund kaum von der Norm ab. Lange Zeit bleibt die Lungenfunktion uneingeschränkt.

Im Laufe von Jahren oder sogar Jahrzehnten tritt durch Zunahme der Mikrolithen und Ossifikation der interlobulären Septen eine interstitielle Fibrose der befallenen Lungenpartien und eine kompensatorische Lungenspitzendehnung auf. Durch diese Umwandlung kommt es zu einer respiratorischen Insuffizienz mit zunehmender Überlastung der rechten Herzkammer, wie bereits bei den ersten postmortalen Fällen beschrieben wurde. Es bestanden Dyspnoe, Zyanose, Trommelschlegelfinger, Uhrglasnägel und gelegentlich Hämopty- sen. Die Dehnung der Lungenspitzen führt zu einer Neigung zum Spontanpneumothorax. Der Serum-Calcium-Spiegel liegt im Normbereich.

\section{Therapie}

Da die Ätiologie dieser Krankheit unbekannt ist, existiert keine kausale Therapie.

Im fortgeschrittenen Stadium ist die Lungentransplantation die Methode der Wahl [6].

\section{Ergebnis}

Bei einem 50-jährigen Patienten wurden bei einer Routineröntgenthoraxuntersuchung multiple kleine Noduli in den Mittel- und Unterfeldern festgestellt. Die Diagnose einer pulmonalen alveolären Mikrolithiasis wurde nach transbronchialer Probeexzision histologisch gesichert. Bei regelmäßigen Kontrollen im Verlauf von 4 Jahren blieb der Befund unverändert.

\section{Diskussion}

Auch ein langjähriger unveränderter röntgenologischer und klinischer Befund der pulmonalen alveolären Mikrolithiasis verlangt eine regelmäßige weitere Kontrolle, um wegen der zu erwartenden Zunahme des Befundes rechtzeitig eine Lungentransplantation zu planen.

\section{Literatur}

${ }^{1}$ Hotchi M, Nasu T, Mihara H. An autopsy case of microlithiasis alveolaris pulmonum - with special attention to elastosis in lung tissue -. Acta Path 1970; 20: 111 - 125

${ }^{2}$ Ucan ES, Keyf AI, Aydilek R, Yalcin Z, Sebit S, Kudu M, Ok U. Pulmonary alveolar microlithiasis: review of turkish reports. Thorax 1993; 48: 171 - 173

${ }^{3}$ Portnoy LM, Amadeo B, Hennigar GR. Pulmonary alveolar microlithiasis. Am J Clin Pathol 1964; 41: 194 - 201

${ }^{4}$ Kent G, Gilbert ES, Meyer HH. Pulmonary microlithiasis. A M A Arch Path 1955; 60: 556-562

${ }^{5}$ Sears MR, Chang AR, Taylor AJ. Pulmonary alveolar microlithiasis. Thorax 1971; 26: 704-711

${ }^{6}$ Stamatis G, Zerkowski HR, Doetsch N, Greschuchna D, Konietzko $\mathrm{N}$, Reidemeister JC. Sequential bilateral lung transplantation for pulmonary alveolar microlithiasis. Ann Thorac Surg 1993; 56: 972-975

${ }^{7}$ Mikhailov V. Pulmolithiasis endalveolaris et interstitialis diffusa. Klin Med (Moskau) 1954; 32: 31 - 36 Zit. in Caffrey und Mitarb.

8 Prakash UBS, Barham SS, Rosenow EC, Brown ML, Spencer Payne W. Pulmonary alveolar microlithiasis. A review including ultrastructural and pulmonary function studies. Mayo Clin Proc 1983; $58: 290-300$

${ }^{9}$ Pankow W, Bittinger A, Adler G, Krüger A, von Wichert P. Microlithiasis alveolaris pulmonum, Kasuistik und Übersicht. Pathologe 1989; 10: 186-193

${ }^{10}$ Caffrey PR, Altman RS. Pulmonary alveolar microlithiasis occurring in premature twins. J Pediatr 1965; 26: 758 - 763

${ }^{11}$ Harbitz F. Extensive calcification of the lungs as a distinct disease. Arch Int Med 1918; 21: 139-146

12 Puhr L. Mikrolithiasis alveolaris pulmonum. Virch Arch 1933; 290: $156-160$ 
${ }^{13}$ Landes G, Leicher F. Kasuistik. Zum Krankheitsbild der Mikrolithiasis alveolaris pulmonum. Ärztl Wschr 1948; 3: 692-695

${ }^{14}$ Lindig W. Ein klinisch-röntgenologischer Beitrag zum Krankheitsbild der „Mikrolithiasis alveolaris pulmonum“. Röfo 1951; 75: $678-684$

15 Petrányi GY, Zsebök Z. Mikrolithiasis alveolaris miliaris pulmonum. Radiologia Clinica (Basel) 1954; 23: 202 - 209

${ }^{16}$ Sosman MC, Dodd GD, Jones WD, Pillmore GU. The familiar occurrence of pulmonary alveolar microlithiasis. A J R 1957; 77: $947-1012$

\section{Dr. med. H. Gubbawy}

Praxis für Lungen- und Bronchialheilkunde Großflecken 41

24534 Neumünster

\section{BUCHBESPRECHUNG}

Russell B., Rayman (Ed.) Clinical Aviation Medicine (Klinische Luftfahrtmedizin). 3 rd. Ed. 346 S., Index. Gebunden; Castle Connolly Graduate Medical Publishing, LLC, New York 2000. ISBN 1-883769-86-8

Als die großen Lehrbücher der Luftfahrt- bzw. der Luft- und Raumfahrtmedizin gelten bis heute in den USA 1. „German Aviation Medicine World War II" (Department of the Air Force, Washington, D. C., 1951), verfasst, mit 45 deutschen Wissenschaftlern, von Hubertus Strughold: die Bibel der „Residents in Aerospace Medicine (RAMs)“ der USAFSAM; 2. „Aerospace Medicine" des legendären Harry G. Armstrong (1961; Zweitauflage von Hugh W. Randel 1971) und 3. die opulenten „Fundamentals of Aerospace Medicine“ von Roy L. DeHart (1985; Zweitauflage 1991).

Für den praktisch tätigen Fliegerarzt - zivil: „Aeromedical Examiner (AME)“ der F. A. A.; militärisch: „Flight Surgeon (FS)“ von USA, USN und USAF - hatte Russell B. Rayman (ehemaliger USNFS) bereits 1982 das handliche Kompendium „Clinical Aviation Medicine“ verfasst (Zweitauflage 1990), das das multidisziplinäre Fach der Flugmedizin kurz und umfassend behandelt; es hat sich im fliegerärztlichen Alltag über die Jahre vielfältig bewährt.

Ergänzt durch die jüngsten Erkenntnisse und Erfahrungen in Medizin, Ergonomie und Flugtechnik liegt nun die überarbeitete, in einigen Kapiteln neu geschriebene dritte Auflage vor - in neuer Gliederung, in größerem Format und Drucksatz, aber ohne den bisherigen Rahmen zu sprengen. Kein Taschenbuch zwar, aber ein „griffiges“ reines Textbuch.

Unter den operationellen Kriterien für Auswahl, Tauglichkeit und Einsatz der „Flieger“ (zivil/militärisch) behandeln Verf. und seine drei Mitarbeiter präventivmedizinische und klinische Probleme. Übergeordnetes Ziel ist es, nur den geistig, psychisch und physisch Geeigneten zur fliegerischen Ausbildung und dann als lizensierten Piloten zuzulassen, die Gesundheit des Fliegers zu überwachen und zu erhalten. Zugleich - unter Berücksichtigung der Flugsicherheit - sind pathophysiologische Befunde wie Erkrankungen und Krankheiten zu bewerten, um über die „Fliegertauglichkeit“, damit auch über zeitlich befristete oder dauernde Flugunfähigkeit zu entscheiden.

In übersichtlicher Gliederung werden die flugmedizinischen Kriterien entsprechend den (nationalen/internationalen) Tauglichkeitsanforderungen (aviation medical standards) in 11 Kapiteln behandelt: 1. Einführung (mit Definitionen der gebräuchlichen Terminologie in der Luftfahrt), 2. Innere Medizin (u.a. Angiologie, Endokrinologie, Gastroenterologie, Hämatologie, Infektionskrankheiten, Nephro-Urologie, Pneumologie, Rheumatologie - und Alkoholismus!), 3. Orthopädie, 4. Neurologie, (John D. Hastings), 5. Ophthalmologie, 6. Otorhinolaryngologie, 7. Kardiologie (William B. Kruyer) 8. Urologie, 9. Dermatologie, 10. Psychiatrie, (Richard A. Levy), 11. Onkologie.

Jedem Kapitel sind ausführliche Literaturhinweise angefügt; ein umfassendes Stichwortverzeichnis (Index) rundet das Werk ab.

Wo erforderlich (und möglich) sind in den einzelnen Kapiteln auch die therapeutischen Möglichkeiten skizziert. Dagegen sind die bei dem weltweiten Luftverkehr heute zunehmenden Erkrankungsgefahren, insbesondere die Tropenkrankheiten und deren vielfältige „getarnten“ Symptome nicht erwähnt worden; sie sollten bei einer Neuauflage in einem eigenen Kapitel Beachtung finden.
H. S. Fuchs, Bad Godesberg 